\title{
Quantum Suppression of Shot Noise in Atom-Size Metallic Contacts
}

\author{
H. E. van den Brom and J. M. van Ruitenbeek \\ Kamerlingh Onnes Laboratorium, Leiden University, Postbus 9504, 2300 RA Leiden, The Netherlands
}

(Received 22 June 1998)

\begin{abstract}
The transmission of conductance modes in atom-size gold contacts is investigated by simultaneously measuring conductance and shot noise. The results give unambiguous evidence that the current in the smallest gold contacts is mostly carried by nearly fully transmitted modes. In particular, for a singleatom contact the contribution of additional modes is only a few percent. In contrast, the trivalent metal aluminum does not show this property. [S0031-9007(99)08411-2]
\end{abstract}

PACS numbers: 72.70.+m, 72.15.Eb, 73.23.Ad, 73.40.Jn

In 1918, Schottky mentioned shot noise as a fundamental shortcoming of vacuum diodes. He realized that the discreteness of electron charge $e$ causes the current to be a Poisson process, and calculated the corresponding mean square current fluctuations to be equal to the product of $e$ and the average current $I$ divided by the total time of averaging [1]. This type of noise is present in all kinds of devices, including microscopic conductors. In the last decade, it has become clear that it can actually be used to obtain information on the electron transport mechanism [2-12]. For example, in a ballistic quantum point contact (QPC) in a two-dimensional electron gas (2DEG), the conductance $G$ as a function of contact diameter shows a stepwise increase by integer multiples of the conductance quantum, $G_{0} \equiv 2 e^{2} / h$ [13]. Recent 2 DEG experiments showed that shot noise was strongly suppressed at quantized conductance values [2,3], in accordance with theoretical predictions [4-8]. In this Letter, measurements of shot noise are performed for the first time to analyze the electronic transport properties of atom-size metallic contacts.

For a metal, the size of an atom is comparable to half the Fermi wavelength $\lambda_{F}$ of the conduction electrons. Therefore, the equivalence of electronic properties of QPCs in a 2DEG and in a metal is far from trivial. In particular, it inhibits a direct observation of the effect of the formation of discrete electron modes in a metallic QPC, i.e., quantization of the conductance. In fact, using a combined scanning tunneling microscopy and atomic force microscopy setup [14] it has been shown that steps in the conductance, observed when stretching the contact, are the result of atomic rearrangements (see also [15]). Primarily, evidence for quantization of the conductance in metals is derived from histograms of the conductance values, which, for gold [16] and sodium [17], show peaks close to integer multiples of $G_{0}$. However, this evidence is not unambiguous, as demonstrated for aluminum, which shows clear peaks near quantized conductance values in the histograms [18], while up to three modes contribute to the conductance near $G=G_{0}$ [19].

In a ballistic QPC with perfect transmission of electrons there are no fluctuations in the occupation numbers of left and right moving electrons, suppressing all shot noise [4-8]. For a contact of size comparable to the Fermi wavelength $\lambda_{F}$, electron transport is described using the Landauer-Büttiker formalism. In this formalism, the shot noise spectral density can be expressed in terms of the transmission probabilities $T_{n}$ of the conducting channels [5],

$$
P_{I}=2 e V \frac{2 e^{2}}{h} \sum_{n} T_{n}\left(1-T_{n}\right)
$$

where the sum is over all modes which fulfill the quantization condition set by the boundaries of the point contact. The label $I$ indicates that we consider current fluctuations $\left\langle\Delta I^{2}\right\rangle$. From Eq. (1) we see that, for a mode with a transmission probability $T_{n}$ close to 1 , the shot noise is indeed suppressed. If all $T_{n}$ are small, the classical full shot noise formula, $P_{I}=2 e I$, is recovered. Including the thermal excitations of quasiparticles for finite temperatures leads to the following expression $[7,8]$ :

$$
\begin{aligned}
P_{I}= & 2 e V \operatorname{coth}\left(\frac{e V}{2 k T}\right) \frac{2 e^{2}}{h} \sum_{n} T_{n}\left(1-T_{n}\right) \\
& +4 k T \frac{2 e^{2}}{h} \sum_{n} T_{n}^{2} .
\end{aligned}
$$

In equilibrium (i.e., $V=0$ ) or in the high temperature limit, the Johnson-Nyquist thermal noise, $4 k T G$, is recovered. For zero temperature, Eq. (2) reduces to the shot noise formula (1). In the experiments described below, we are interested in the amount of noise above the thermal noise, which we will call excess noise, $P^{\operatorname{exc}}(I) \equiv$ $P(I, T)-P(0, T)$.

In order to obtain a stable atomic scale contact, we use the mechanically controllable break-junction technique. A notched, 99.998\% pure, gold wire is glued on top of a phosphor bronze substrate, which is insulated with kapton foil. This is mounted into a vacuum can and cooled down to $4.2 \mathrm{~K}$. By bending the substrate, the wire is broken, after which contact between the fracture surfaces is controlled using a piezoelectric element. For a more complete description of the technique, see Ref. [20]. 
The noise level we are expecting is on the order of $n V / \sqrt{\mathrm{Hz}}$. In order to measure such small signals, we use the experimental setup schematically drawn in Fig. 1. The signal to be measured is first amplified $10^{5}$ times by two stages of low noise wide band preamplifiers (dc-1 MHz and $0.5 \mathrm{~Hz}-1 \mathrm{MHz}$, respectively). In order to suppress the noise of the preamplifiers, we use two sets of preamplifiers in parallel and feed the signals into a spectrum analyzer, which calculates the crosscorrelation Fourier spectrum. The conductance of the sample is measured using the dc voltage after the first amplifier; the bias current is sent using a battery ( 0 $9 \mathrm{~V})$ with a large series resistance, the latter being at $4.2 \mathrm{~K}$ close to the sample. Since we are interested in contacts with resistance around $13 \mathrm{k} \Omega$, and our wiring has a capacitance of around $250 \mathrm{pF}$, this introduces a low pass filter with a cutoff frequency of around $50 \mathrm{kHz}$. To correct the measured spectra, $P_{V}(I)$, for the electronic transfer function of the system, we send white noise, $P_{V}^{\text {src }}$, from a calibration source through a $1 \Omega$ series resistance and measure the frequency response of the setup $P_{V}^{\mathrm{cal}}$. The excess noise then equals

$$
P_{V}^{\mathrm{exc}}(I)=\frac{P_{V}(I)-P_{V}(0)}{P_{V}^{\mathrm{cal}}-P_{V}(0)} \times P_{V}^{\mathrm{src}} .
$$

The label $V$ indicates that here we consider the spectral density of voltage fluctuations $\left\langle\Delta V^{2}\right\rangle$ [21].

Obtaining one single spectrum in the interval $250 \mathrm{~Hz}-$ $100 \mathrm{kHz}$ and averaging 10000 times takes about $1 \mathrm{~min}$. For each contact setting, we have to take several spectra: one for calibration, one for thermal noise, and several with different dc current biases. During this procedure, the contact is verified to be stable by measuring the conductance between each two spectra. Measuring the conductance twice with different polarity of the bias current eliminates dc offset of the preamplifiers.

For frequencies smaller than the inverse dephasing time and obeying $h f \ll e V, k T$ the spectrum of both thermal and shot noise is white, i.e., frequency independent. This is not the case for so-called $1 / f$ noise, which is generally believed to be a consequence of defect motion and

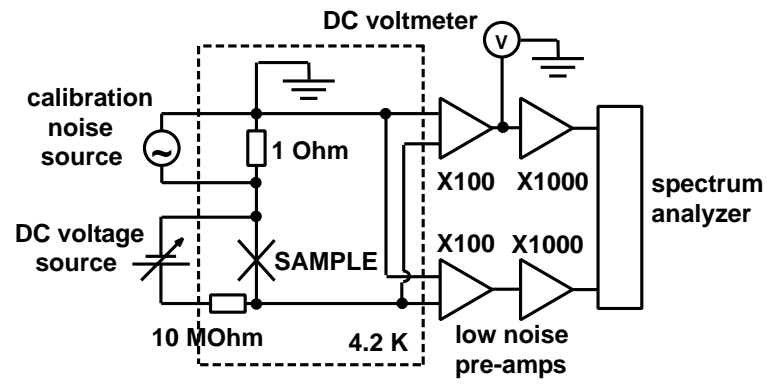

FIG. 1. Wiring diagram of the experimental setup for shot noise measurements on atom-size point contacts. The area enclosed by the dashed lines indicates the part at $4.2 \mathrm{~K}$. decreases at higher frequencies as $f^{-1}$. External vibrations (e.g., sound) and pickup of external electromagnetic signals can be recognized in the Fourier spectrum. External vibrations are mostly visible at low frequencies, in the same range where $1 / f$ noise dominates, which is why we concentrate on the higher frequency part of the spectrum. Electromagnetic pickup, present despite careful shielding, is seen as sharp peaks and is removed when we take the difference (3). In addition, the preamplifier noise left over after taking the cross correlation is further removed this way. Furthermore, the effect of external vibrations as well as $1 / f$ noise is quadratic in the supplied current, while shot noise is linear. We verify that the excess noise we measure has the proper current dependence.

A typical example of the raw data, $P_{V}(I)$, we obtain can be seen in Fig. 2a, where we show measurements on a contact of $8.4 \mathrm{k} \Omega$, or $1.53 G_{0}$. The zero bias noise at low frequencies is in agreement with the expected thermal noise, $4 k T R$. At higher frequencies, we observe a rolloff, which is due to the frequency response of the setup. When applying a bias current the noise level increases. Apart from an increase in noise level over the entire spectrum, we see a rapid rise at low frequencies, which we attribute to $1 / f$ noise and external vibrations. The excess noise, $P_{V}^{\mathrm{exc}}(I)$, corrected for the transfer function according to Eq. (3), is plotted in Fig. 2b and approaches a white spectrum for higher frequencies. At the highest frequencies, a small decrease is observed due to higher order stray capacitance corrections, which we take into account in the error bars of Figs. 3 and 4. To show that we are indeed measuring shot noise, we investigated the current dependence and verified the expected behavior given by Eq. (2), which is nearly linear for higher current: In Fig. 3, we compare the data for a contact with $G=$ $1.02 G_{0}$ to full shot noise, $2 e I$, and to Eq. (2) with
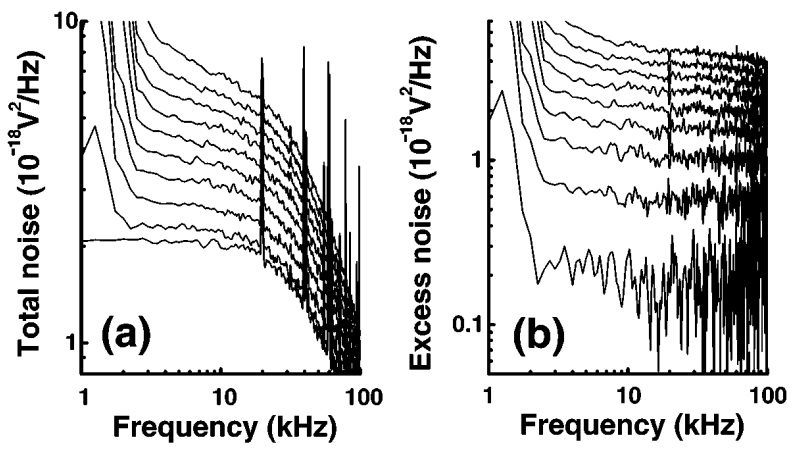

FIG. 2. (a) Raw data for a gold contact of $1.53 G_{0}$ at $4.2 \mathrm{~K}$, without correction for the electronic transfer of the setup. The lowest curve shows the thermal noise, a few percent higher than the theoretical value $2.0 \times 10^{-18} \mathrm{~V}^{2}$, which is due to the preamplifier noise. The upper curves are the total measured noise for increasing current, $I=0.1,0.2,0.3, \ldots, 0.9 \mu \mathrm{A}$. (b) Excess noise, calculated from the data in (a) and corrected according to Eq. (3). Note that the effect of mechanical vibrations is visible at lower frequencies. 


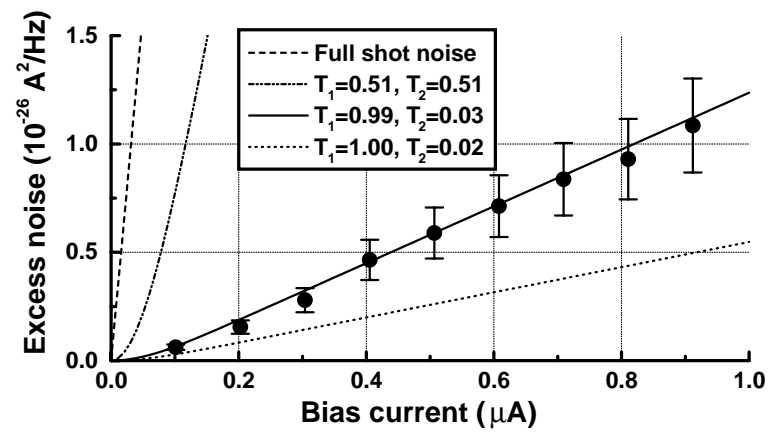

FIG. 3. Measured excess noise values for a contact with $G=1.02 G_{0}$, as a function of bias current. For comparison we plot full shot noise, $2 e I$, and Eq. (2) for the case of one single partially transmitted wave (i.e., $T_{1}=1$ and $T_{2}=0.02$ ) and for the case of two equally transmitted waves $\left(T_{1}=T_{2}=0.51\right)$. A good description of the data is obtained with $T_{1}=0.99$ and $T_{2}=0.03$.

different combinations of transmission values. As can be seen in the figure, the data are well described by one almost fully transmitted mode together with one mode with a very small transmission probability.

A number of contacts with conductances in the range $(0.7-4.1) G_{0}$ have been similarly analyzed. In Fig. 4, we plot the excess noise values corresponding to the measured voltage noise at maximum bias current $(0.9 \mu \mathrm{A})$ as a function of conductance for 27 different contacts. The figure shows that all values are small, compared to $2 e I$. A smallest value of $(0.02 \pm 0.005) 2 e I$ is observed for a contact with conductance very near $G_{0}$. For comparison, we show the expected behavior, based on Eq. (2), when the conductance is due to only fully trans-

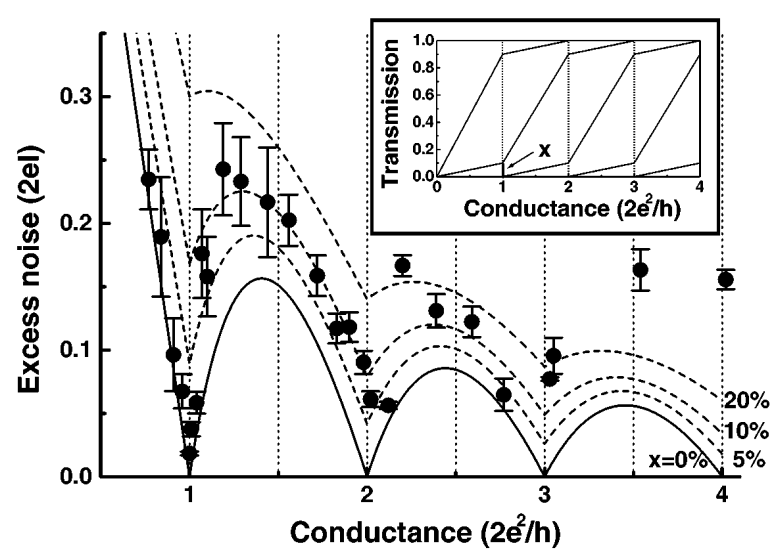

FIG. 4. Measured excess noise values for 27 gold contacts at $4.2 \mathrm{~K}$ with a bias current of $0.9 \mu \mathrm{A}$. Comparison is made with calculations in the case of one single partially transmitted mode (full curve) and for various amounts of contributions of other modes according to the model described in the inset (dashed curves). In the limit of zero conductance, these curves all converge to full shot noise, i.e., $2.9 \times 10^{-25} \mathrm{~A}^{2} / \mathrm{Hz}$. Inset: transmission of modes in the case of $x=10 \%$ contribution from neighboring modes. mitted modes $\left(T_{n}=1\right)$ plus a single partially transmitted mode (full curve). All measured points are at or above this curve, as is expected. From our measurements we cannot determine the complete set $\left\{T_{n}\right\}$, since we have only two measured parameters (i.e., conductance and shot noise), and we can obtain at most two independent parameters $T_{n}$. To visualize the effect of contributions of different modes to the conductance, we use the model described in the inset of Fig. 4. In this model the conductance between $(n-1) G_{0}$ and $n G_{0}$ is built up as $G=(n-2) G_{0}+\left(T_{n-1}+T_{n}+T_{n+1}\right) G_{0}$, where the three partially open channels have transmissions which increase linearly, and the sum of $\left(1-T_{n-1}\right)$ and $T_{n+1}$ is a constant fraction $x$. This model has no physical basis but serves merely to illustrate the extent to which additional, partially open channels are required to describe the measured shot noise. The corresponding behavior of the excess noise as a function of conductance, calculated from Eq. (2), is shown as the dashed curves in Fig. 4 for $x=5 \%, 10 \%$, and 20\%. We see that for $G<G_{0}$ the data are very close to the $x=0 \%$ curve, while for $G_{0}<G<2 G_{0}$ the data are closer to the $x=10 \%$ curve. In particular, the minimum at $G=G_{0}$ is very sharp, while also a minimum near $G=2 G_{0}$ is visible. For $G>2 G_{0}$, the contribution of other partially open channels continues to grow. Each point is measured for a different contact, and the contribution of modes to the conductance of each contact can, in principle, be widely different. For example, just above $2 G_{0}$ we find a point with $x<5 \%$, while the next point has $x>20 \%$. Hence, the scatter in the data compared to any of the curves is not due to statistical errors but is a result of the intrinsic variation in the properties of the contact.

It should be stressed that the results described above are obtained in a gold QPC. We performed similar experiments on aluminum, which showed much weaker suppression of shot noise. For Al contacts between $0.8 G_{0}$ and $2.5 G_{0}$ the obtained shot noise values vary from 0.3 to $0.6(2 e I)$. These values fit the theory only if one assumes contributions from a much wider set of modes. In particular, the conductance at $G=G_{0}$ is not carried by one fully transmitted mode but by at least two partially transmitted modes instead.

In model simulations for gold, Brandbyge et al. [22] find nearly full transmission of the channels for $G$ near 1 and $3 G_{0}$. However, they report two half-opened channels around $G=2 G_{0}$, which is at variance with the present experimental results.

For atomic-size contacts of superconductors, Scheer et al. [19,23] performed experiments on current-voltage characteristics in the superconducting state. This was used to demonstrate that the number of conducting channels in a single atom contact is determined by the number of valence orbitals [23,24]. However, reservations were made about the results on gold, since the proximity effect was used to induce superconductivity in a gold 
QPC, causing a modified quasiparticle density of states. Our results for $G<G_{0}$ show unambiguously that, in the monovalent metal gold, the current through a single atom contact is indeed almost exclusively carried by one single conductance channel. Our results for a single atom contact of aluminum (which has a valency of three) confirm that several modes are transmitted $[19,23,24]$. In addition, for gold we find that the conductance for larger contacts is well-described by a set of conductance channels, where all are fully open, except one, which carries the fraction of conductance above the integer value. This property was also inferred from measurements of conductance fluctuations [25] and thermopower [26] by Ludoph et al., and was referred to as "saturation of channel transmission." From Fig. 4, we can even quantify the accuracy to which this rule is obeyed. Where the interpretation of Refs. [25,26] depends on ensemble averaged properties, the present results show that saturation of channel transmission is observed for individual contacts, and is independent of any adjustable parameters.

From conductance histograms, the quantum conductance properties cannot be identified as unambiguously in metals [18] as they are in 2DEG contacts. In gold QPCs, the conductance does not show the pronounced preference for integer multiples of $G_{0}[16,27]$ as would be expected for truly quantized conductance. In contrast, the quantum suppression of shot noise observed here is even more pronounced than in 2DEG systems [2,3]. By this method the quantum nature of the conductance in atom-size metallic point contacts is now straightforwardly revealed.

This work is part of the research program of the Stichting voor Fundamenteel Onderzoek der Materie (FOM), which is financially supported by NWO. We thank D. C. Glattli, J. I. Dijkhuis, and M. G. Peters for helpful discussions and L. J. de Jongh for his stimulating interest in this work.

[1] W. Schottky, Ann. Phys. (Leipzig) 57, 541 (1918).
[2] M. Reznikov et al., Phys. Rev. Lett. 75, 3340 (1995).

[3] A. Kumar et al., Phys. Rev. Lett. 76, 2778 (1996).

[4] G. B. Lesovik, Sov. Phys. JETP Lett. 49, 592 (1989).

[5] M. Büttiker, Phys. Rev. Lett. 65, 2901 (1990).

[6] C. W. J. Beenakker and H. Van Houten, Phys. Rev. B 43, 12066 (1991).

[7] Th. Martin and R. Landauer, Phys. Rev. B 45, 1742 (1992).

[8] M. Büttiker, Phys. Rev. B 46, 12485 (1992).

[9] C. W. J. Beenakker and M. Büttiker, Phys. Rev. B 46, 1889 (1992); M. J. M. de Jong and C. W. J. Beenakker, Phys. Rev. B 46, 13400 (1992); K. E. Nagaev, Phys. Lett. A 169, 103 (1992).

[10] F. Liefrink et al., Phys. Rev. B 49, 14066 (1994).

[11] A. H. Steinbach, J. M. Martinis, and M. H. Devoret, Phys. Rev. Lett. 76, 3806 (1996).

[12] L. Saminadayar et al., Phys. Rev. Lett. 79, 2526 (1997).

[13] B. J. van Wees et al., Phys. Rev. Lett. 60, 848 (1988); D. A. Wharam et al., J. Phys. C 21, L209 (1988).

[14] G. Rubio, N. Agraït, and S. Vieira, Phys. Rev. Lett. 76, 2302 (1996).

[15] H.E. van den Brom, A.I. Yanson, and J. M. van Ruitenbeek, Physica (Amsterdam) 252B, 69 (1998).

[16] M. Brandbyge et al., Phys. Rev. B 52, 8499 (1995).

[17] J. M. Krans et al., Nature (London) 375, 767 (1995).

[18] A. I. Yanson and J. M. van Ruitenbeek, Phys. Rev. Lett. 79, 2157 (1997).

[19] E. Scheer et al., Phys. Rev. Lett. 78, 3535 (1997).

[20] C. J. Muller, J. M. van Ruitenbeek, and L. J. de Jongh, Physica (Amsterdam) 191C, 485 (1992); Phys. Rev. Lett. 69, 140 (1992).

[21] The relation between current and voltage noise is simply $P_{V}=P_{I} / G^{2}$.

[22] M. Brandbyge, M. R. Sørensen, and K. W. Jacobsen, Phys. Rev. B 56, 14956 (1997).

[23] E. Scheer et al., Nature (London) 394, 154 (1998).

[24] J. C. Cuevas, A. Levy Yeyati, and A. Martín-Rodero, Phys. Rev. Lett. 80, 1066 (1998).

[25] B. Ludoph, D. Esteve, C. Urbina, M. H. Devoret, and J. M. van Ruitenbeek, following Letter, Phys. Rev. Lett. 82, 1530 (1999).

[26] B. Ludoph and J. M. van Ruitenbeek (to be published).

[27] Z. Gai et al., Phys. Rev. B 53, 1042 (1996). 